\title{
Energy loss for evaluating heart valve performance
}

\author{
Cary W. Akins, MD, ${ }^{a}$ Brandon Travis, $\mathrm{PhD},{ }^{\mathrm{b}}$ and Ajit P. Yoganathan, $\mathrm{PhD}^{\mathrm{c}}$
}

Energy loss is a well-established engineering concept that when applied to evaluating the performance of native heart valves and valvular prostheses has the potential for providing valuable information about the impact of valve function on myocardial performance. The concept has been understood for many years, but its routine application has been hindered not only by a lack of understanding of its meaning but also because of the lack of investigational tools to easily obtain the data necessary for its estimation. Today the gathering of that information is becoming easier, and thus the time has come to revisit the efficacy of energy loss for evaluating heart valve performance. This review defines what energy loss is, how it is measured, and how it might be applied to clinical situations of heart valve disease to better understand the impact of valvular disease on ventricular function.

From the Division of Cardiac Surgery, Massachusetts General Hospital, Boston, Mass, ${ }^{\text {a }}$ T-Forskning, Skejby Sygehus, Brendstrupgaardsvej, 8200 Árhus N, Denmark, ${ }^{\mathrm{b}}$ and Wallace H. Coulter School of Biomedical Engineering, Georgia Institute of Technology and Emory University, Atlanta, $\mathrm{Ga}^{\mathrm{c}}$

Received for publication Aug 14, 2007; revisions received Dec 4, 2007; accepted for publication Dec 18, 2007.

Address for reprints: Cary W. Akins, MD, Department of Surgery, Cox 648, Massachusetts General Hospital, 55 Fruit Street, Boston, MA 02114 (E-mail: cakins@partners.org).

J Thorac Cardiovasc Surg 2008;136:820-33 0022-5223/\$34.00

Copyright $(\underset{2008}{ } 20 y$ The American Association for Thoracic Surgery

doi:10.1016/j.jtcvs.2007.12.059
$\mathrm{E}$ ven though in the early 1950s Gorlin and Gorlin ${ }^{1}$ developed an equation using data obtained from cardiac catheterization findings that allowed the calculation of the functional valve area of a diseased native heart valve, the focus of assessing diseased valves for many clinicians remained the peak gradient across the valve.

After the first successful mitral valve replacement by Starr, reported by Starr and Edwards, ${ }^{2}$ and orthotopic aortic valve replacement by Harken and associates, ${ }^{3}$ both in 1960 , clinicians were merely pleased that a prosthetic heart valve was substantially better than the very diseased native valve it replaced. Little attention was initially paid to the hemodynamic performance of the first caged ball prostheses, which provided at least moderate gradient relief and essentially no regurgitation once the ball was seated.

Not until there came the realization that the initial prostheses could be improved and competitive prostheses became available did there evolve an interest in assessing and promoting the hemodynamic performance of prostheses. In the 1960s Edwards first used peak gradient to describe the performance of the Starr-Edwards valvular prosthesis. Over the course of the next several decades, particularly after echocardiography became an accepted clinical tool, investigators and clinicians gradually refined the assessment of valve performance, albeit concentrating principally on the systolic performance of aortic prostheses and the diastolic performance of mitral prostheses, as though valvular regurgitation were of little importance.

Peak gradients gave way to mean gradients. Mean gradients were in turn overshadowed by valve areas. Valve areas were then indexed to body size and finally measured in vivo to yield indexed effective orifice areas. Yet none of these accounts for valvular regurgitation, nor do they accurately represent prosthetic performance in all specific clinical settings.

In 1987 Leefe and Gentle ${ }^{4}$ wrote about energy loss as it related to native and prosthetic valve performance. The concept could account for some of the shortcomings of previous valve performance assessments, but the information necessary to calculate energy loss was not clinically easy to obtain. They stated in that publication, "the full potential of the energy loss method has not yet been fully realized." We believe that statement still holds true.

Today, with sophisticated echocardiographic techniques the accumulation of the necessary data to estimate energy loss is much easier; yet the concept remains underused and generally not well understood. The purpose of this presentation is to describe what energy loss means, how it is measured, and how it might be of help to clinicians 
interested in a more complete and accurate assessment of native and prosthetic valve performance. The main body of the presentation will contain limited mathematical equations used to define the concepts discussed. For those so inclined, the Appendix will contain a more thorough review of the supporting mathematics.

\section{Energy Loss as a Concept}

Energy loss not only allows an assessment of the forward performance of a heart valve or prosthesis, but also can account for any regurgitation. The concept can allow for differing levels of performance depending on the clinical or anatomic situation in which the valve or prosthesis performs. Energy loss allows the investigator to venture beyond the mere raw assessment of a mechanical device and move forward to assess the impact of valve performance on cardiac function. Drawing an analogy from another area of cardiac disease, energy loss would be comparable to assessing the impact of a specific coronary artery lesion on myocardial performance, instead of merely measuring the degree or length of a coronary stenosis. Potentially, measurement of energy loss in patients with concomitant aortic and mitral valve lesions, such as aortic stenosis and mitral regurgitation, may lead to a more complete understanding of the impact of the combined pathologic conditions on left ventricular function.

As a further analogy, let us look at the various determinations of valve performance as compared to increasingly sophisticated ways of looking at the performance of an automobile engine (Table 1).

Only in the last assessment of each device does one have the true representation of the impact of the valve or automobile engine on the performance of the heart or vehicle.

To place into perspective the potential value of energy loss as a means of evaluating patients, for example, those with aortic stenosis, consider that one patient with a very high systolic gradient across the aortic valve may have no symptoms, while another patient with an identical gradient may be very symptomatic. Indeed, angina pectoris and left ventricular failure in patients with severe aortic stenosis are verified predictors of an increased risk of sudden death. Thus valve area itself is not the sole predictor of symptoms or, by probable logical extension, sudden death. As noted by Heinrich and associates, ${ }^{5}$ both symptomatic predictors of sudden death, namely angina pectoris or left ventricular failure, reflect

TABLE 1. Progressive performance criteria

\begin{tabular}{ll}
\hline \multicolumn{1}{c}{ Heart valve } & \multicolumn{1}{c}{ Automobile engine } \\
\hline $\begin{array}{l}\text { Peak gradient } \\
\text { Mean gradient }\end{array}$ & $\begin{array}{l}\text { Number of cylinders } \\
\text { Valve orifice area }\end{array}$ \\
$\begin{array}{l}\text { Indexed effective } \\
\text { orifice area }\end{array}$ & Morsepower \\
Energy loss & Horsepower to weight ratio \\
\hline
\end{tabular}

a pathologic level of left ventricular work. Angina pectoris occurs when myocardial demand for oxygenated blood exceeds supply. Left ventricular failure owing to severe systolic load on the ventricle occurs when the demand for left ventricular work exceeds the intrinsic contractile capacity of the left ventricular myocardium. The assessment of energy loss of the left ventricle resulting from severe aortic stenosis might improve the risk stratification of patients with aortic stenosis, particular those who are clinically asymptomatic, and allow for more appropriate timing of intervention.

\section{Understanding the Determinants of Energy Loss}

Before discussing energy loss to assess heart valve or prosthesis performance, we need to develop some understanding of what energy loss represents and how it is measured.

The following section will define what energy is and describe the various forms energy can take, the sources of energy, and its conversion from one form to another in the cardiovascular system. Finally, the concept of energy loss will be presented-what energy loss is and what it is not.

\section{Mechanical Energy}

Let's begin with what mechanical energy is and its various forms. Basically, mechanical energy is the ability to accelerate a mass of material over a certain distance. Although energy can also be reported in calories or kilowatt-hours, in the setting of evaluating cardiac performance, energy is measured in joules. In its simplest form the definition of 1 joule is as follows:

$$
1 \cdot J=1 \cdot N \cdot m=1 \cdot P a \cdot m^{3}=1 \cdot \frac{\mathrm{kg} \cdot \mathrm{m}^{2}}{\mathrm{~s}^{2}}
$$

where $\mathrm{J}$ represents joules (units of energy), $N$ Newtons (units of force), $m$ meters (units of distance), $P a$ Pascals (units of pressure), $k g$ kilograms (units of mass), and $s$ seconds (units of time).

Equation 1 shows that energy per unit distance has the same dimensions as force, and energy per unit volume the same dimension as pressure. In evaluating cardiac performance, pressure measurement is most commonly used, and because of this, the clearest way to report energy in medicine is on a per unit of volume basis. Hence, this article will report energy per unit volume of blood. However, it is important to remember that the pressure measured by catheter, the static pressure, is only one of several forms of mechanical energy. Although they share the same units, static pressure and total mechanical energy per unit volume are not equivalent.

\section{Forms of Mechanical Energy}

In the circulatory system, mechanical energy can exist in three forms: (1) static pressure, (2) acceleration resulting from gravity, and (3) kinetic energy. Let's look briefly at how each of these forms of energy is represented. 


\section{Pressure}

Pressure $(p)$ is a form of mechanical energy per unit volume that represents a force $\left(F_{p}\right)$ per unit area $(A)$. If a force moves a mass over a distance $(d)$, it performs work, expending pressure energy $\left(E_{p}\right)$. The area that the force acts on multiplied by the same distance represents a volume $(V)$ :

$$
p=\frac{F_{p}}{A}=\frac{F_{p} d}{A d}=\frac{E_{p}}{V}
$$

\section{Acceleration due to gravity}

Acceleration due to gravity $(g$ ) creates another form of mechanical energy per unit volume. A mass $(m)$ accelerating due to gravity creates a force $\left(F_{g}\right)$. If this force moves the mass over a vertical distance $(h)$, it too performs work and expends gravitational energy $\left(E_{g}\right)$. The energy per unit volume is obtained by substituting density $(\rho)$ for mass per unit volume.

$$
\rho g h=\frac{m g h}{V}=\frac{F_{g} h}{V}=\frac{E_{g}}{V}
$$

\section{Kinetic energy}

Mechanical energy per unit volume also can exist in the form of kinetic energy, or energy of movement. If a mass $(m)$ is moving at a velocity $(v)$, it contains kinetic energy, equivalent to half the product of the mass and the square of the velocity:

$$
\frac{1}{2} \rho v^{2}=\frac{\frac{1}{2} m v^{2}}{V}=\frac{E_{k}}{V}
$$

Again, the energy per unit volume is obtained by substituting density for mass per unit volume.

\section{Conversion of Mechanical Energy}

Pressure, gravitational, and kinetic energies in the circulation can be freely converted from one form to another without energy loss. This concept is illustrated by Bernoulli's equation, which relates the relative amounts of pressure, gravitational, and kinetic energy per unit volume between two spatial locations along a path in the flow, assuming no energy is lost.

$$
\frac{1}{2} \rho v_{1}^{2}+\rho g h_{1}+p_{1}=\frac{1}{2} \rho v_{2}^{2}+\rho g h_{2}+p_{2}(\text { Bernoulli equation })
$$

where ${ }^{1} / 2 \rho \nu^{2}$ is kinetic energy, $\rho g h$ is acceleration owing to gravity, and $p$ is pressure at locations 1 and 2 in the circulation.

Bernoulli's equation for steady flow states the total mechanical energy per unit volume at two spatial locations is the same but can exist in different forms. For example, a decrease in pressure from one location to another may be balanced by an increase in either fluid velocity or height without loss of total energy. A pressure drop measured between two locations is, therefore, not mechanical energy loss if it is accompanied by increases in either gravitational or kinetic energy. These energies can be converted back to pressure energy later. (See Appendix Equations A4 and A5.)

\section{Source of Mechanical Energy}

The primary source of mechanical energy in the circulation is work performed by the left ventricle. Ventricular contraction generates mechanical energy in blood in the form of static pressure. Conversion of pressure to kinetic energy leads to movement of a volume of blood.

The energy generated by the ventricle during one cardiac cycle is depicted in a pressure-volume diagram (Figure 1), which shows changes in pressure and volume in the ventricle during one cardiac cycle. The cycle begins with the period of diastolic filling, when the mitral valve is open and the ventricle fills (Figure 1 -curve between points 1 and 2 ). When the mitral valve closes, the period of isovolumic contraction begins. For a period of time the volume in the ventricle remains constant, but the pressure rises rapidly (Figure 1-curve between points 2 and 3 ). When the aortic valve opens, starting the period of systolic ejection, blood moves out of the ventricle into the ascending aorta (Figure 1 - curve between points 3 and 4). When the aortic valve closes, the period of isovolumic relaxation begins (Figure 1 -curve between points 4 and 1). During this period before the mitral valve opens, the ventricular volume again remains constant, but the pressure falls, returning to the start of the next cycle.

The energy generated by the ventricle during one cardiac cycle is equivalent to the integral of the pressure-volume diagram (Figure 1, shaded area). The energy per unit volume

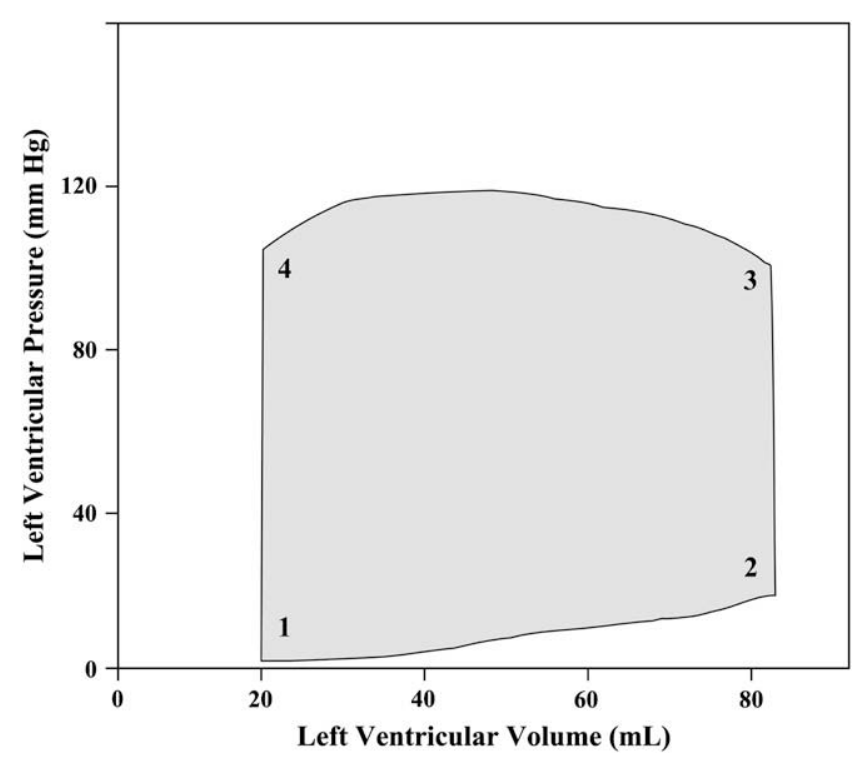

Figure 1. Pressure-volume diagram of one cardiac cycle. 
generated by the ventricle is equivalent to the integral of the pressure diagram divided by the stroke volume. This is roughly equivalent to the average increase in left ventricular pressure from diastole to systole. The ventricle thus creates energy in the form of static pressure, and this energy is converted to gravitational and kinetic energy elsewhere in the circulation.

\section{Mechanical Energy Loss}

In addition to its conversion between pressure, gravitational, and kinetic forms, mechanical energy can be converted to heat through friction between moving blood and the stationary vessel walls. Such heat cannot be reconverted to mechanical energy, and thus energy is said to be lost.

Frictional energy losses take one of three forms: viscous losses, turbulence losses, and flow separation losses. All such losses are the result of the fluid interaction with the solid boundary of the vessel wall. (Additional energy can also be effectively lost in the circulation owing to valvular insufficiency and pressure wave reflection.)

\section{Viscous losses}

As a result of frictional forces, fluid immediately adjacent to a solid boundary moves with the same velocity as the boundary. In the case of a stationary vessel, fluid immediately adjacent to the vessel wall does not move, no matter how fast the more central flow is moving. As one moves away from the solid boundary, the velocity of the fluid increases with respect to the radial distance within the vessel (Figure 2). Fluid viscosity, the tendency of fluid components or molecules to "stick" to each other, creates friction between the fluid components in close proximity if they move at different velocities. This is the mechanism of viscous energy loss. Viscous energy losses are proportional to flow rate. Viscous energy loss is unavoidable but can be reduced in vessel flow by slight increases in the radius of the vessel. (This is the reason that vasodilators are helpful in relieving left ventricular workload.) (See Appendix Equations A4-A10.)

\section{Turbulent losses}

Turbulent losses usually do not occur in the healthy circulation, but when they do, they can be of greater magnitude than viscous losses. Turbulence is characterized by random spatial and temporal differences in direction and magnitude of fluid velocity and is the result of the inertia of flow being too great for frictional forces to stabilize fluid movement. A useful analogy to turbulent flow can be demonstrated by the movement of a car. As a car approaches a curve in the road and begins to turn, the car undergoes a change in inertia in the direction of the curve. As long as the car maintains a low velocity relative to the friction that its tires generate to hold it on the road, the car follows the path of movement of its tires. If it does not, the frictional forces cannot hold the car on the road, and it slides across the pavement. This sliding increases the friction between the tires and the road, resulting in large conversion of kinetic energy to heat.

Turbulent flow yields similar results. In a straight vessel, changes in inertia are initiated by small irregularities, or roughness, on the surface of the vessel. If these irregularities become large enough, viscous forces can no longer dampen them, and they propagate changes in inertia elsewhere in the flow (Figure 3). Friction between fluid components in close proximity moving at different velocities eventually converts this random kinetic energy to heat. As in the case of the skidding car, the tendency for turbulence in fluid flow depends on the geometry of the path that the fluid must travel and the ratio of inertial to frictional forces in the flow. In blood flow this ratio is approximated by the Reynolds number. (See Appendix Equation A3.)

Turbulent losses are in general proportional to the square of the flow rate and can be eliminated by reducing the Reynolds number below the value at which the flow transitions into turbulence. Turbulent losses can also be eliminated or reduced by smoothing solid surfaces or by removing sharp corners and bends from the flow geometry, as these can cause flow to destabilize at lower Reynolds numbers. (See Appendix Equations A11-A13.)

\section{Flow separation losses}

Flow separation occurs when slow moving flow near a solid boundary reverses its direction. In the circulation, such reversal occurs either after the passage of the pressure wave from the heart or after the flow has experienced a sudden expansion. In both situations flow experiences deceleration, or decrease in kinetic energy. Bernoulli's equation shows that in the absence of differences in gravitational energy, a decrease in kinetic energy is accompanied by a rise in downstream pressure. If such a rise in pressure occurs, it can overcome

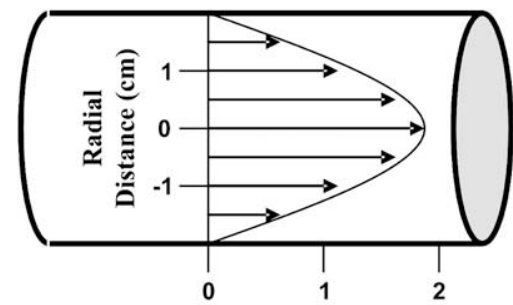

A

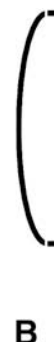

Figure 2. Flow velocity profile under laminar conditions (A) and turbulent conditions (B). 


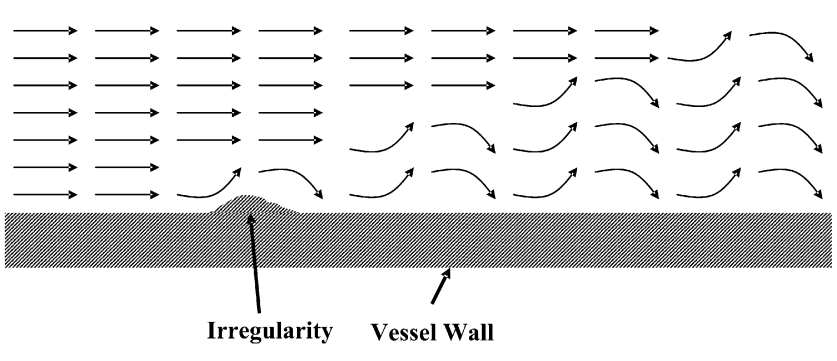

Figure 3. Turbulence resulting from interruption of laminar flow by an irregularity in a vessel wall.

the inertia of the slow moving flow near the vessel wall, reversing its direction. Substantial energy may be required to reinitiate the forward movement of the fluid. This is energy loss resulting from flow separation. Like turbulence, flow separation originates from changes in fluid momentum, and energy loss from separation is generally proportional to the square of the flow rate. Flow separation losses are dependent on the ratio of the diameter of the small orifice where the expansion begins to the diameter of the larger vessel where the expansion is completed. Such losses can be reduced or eliminated by ensuring that all flow expansion is gradual, initiating turbulence in the flow, or orienting oblique obstructions parallel to the incoming flow direction. (See Appendix Equations A14-A21.)

For example, Figure 4, $a$ to $d$, illustrates the impact on flow separation of various shapes inserted into a flow stream. With a spherical ball (Figure 4, $a$ ) there is considerable flow separation. If the ball is converted into a teardrop shape (Figure $4, b$ ), flow separation can be prevented. Gradual expansion allows the low velocity fluid near the vessel wall to mix with the higher velocity fluid closer to the center of the vessel as the pressure gradient increases. This mixing gives forward momentum to the low velocity fluid, preventing its separation. A protrusion on the downstream portion of the ball (Figure 4,c) may initiate turbulence in the flow, reducing the area of flow separation. Turbulence accelerates the rate of mixing between low and higher velocity fluid, permitting more abrupt flow expansion without separation. With a disc shape, typical of a tilting disc valve (Figure $4, d$ ), there is little to no flow separation. Orienting oblique flow obstructions parallel to the incoming flow direction reduces the area over which flow is likely to separate.

\section{Valvular insufficiency}

To compensate for regurgitant flow, the ventricle must reverse the momentum of this flow and pump the fluid again. The additional energy required to perform this action while maintaining the required net forward flow rate represents an important form of energy loss in patients with valvular insufficiency. As can be expected, this form of energy loss is heavily dependent on the regurgitant volume and can be minimized by reducing regurgitant flow.

\section{Pressure wave reflection}

Cardiovascular flow may be slowed by the reflection of the pressure wave from the ventricle back to its source. This mechanism is similar to reflection of light waves as they travel from air through a glass plate. The difference in a property called optical impedance between air and glass causes some of the light waves contacting the glass to be reflected at its surface. The larger the difference in optical impedance between air and glass, the greater the percentage of light waves that are reflected.

Similarly, pressure waves in the circulation may be reflected as they encounter changes in vascular impedance. Most natural changes in vascular impedance are small and occur due to vessel branching. Pathologic changes in vascular impedance, such as those that occur between a healthy and calcified vessel or an aneurysm, can be quite large. Vascular impedance is a function of the size, thickness, and elasticity of the vessel. Pressure wave reflection can be minimized by matching impedance between two adjoining vessels. For this reason, it is advantageous to match the impedance of a vascular graft to that of its adjoining vessels.

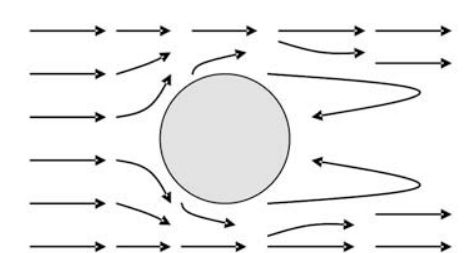

A

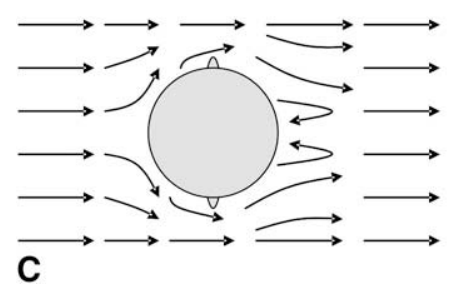

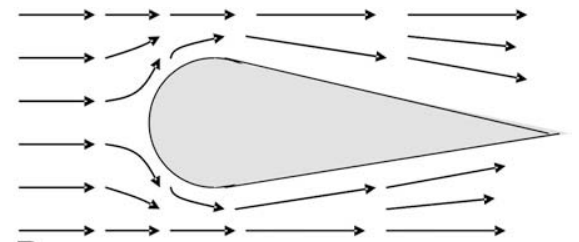

B

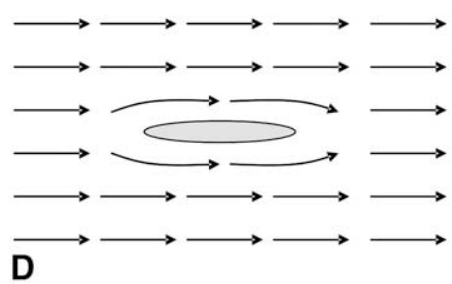

Figure 4. Flow separation created by various shapes inserted into flow stream: $A$, spherical ball; B, teardrop; C, ball with lateral protrusions; D, disc. 
In summary several forms of energy loss exist in the circulation, and the magnitudes of these losses are controlled by different variables. Collectively, they determine the relationship between useful work performed by the left ventricle and net forward flow rate.

\section{Energy Conversion and Loss in a Stenosis}

To put the previous principles into a clinical context, let's consider how mechanical energy may be changed from one form to another, or lost, across a hypothetical arterial stenosis. The area of stenosis can be divided into contraction, throat, and expansion sections (Figure 5).

\section{Contraction section}

The first section of the stenosis is a flow contraction (Figure 5 -point 1 to point 2 ). Because the continuity equation states that velocity downstream must be higher than that upstream, kinetic energy increases across the area of contraction. This increase in kinetic energy occurs at the expense of a large amount of pressure. This conversion of pressure to kinetic energy within the contraction section is the quantity measured when one estimates the pressure gradient with Doppler echocardiography. The Doppler gradient does not, therefore, measure any form of lost driving force for flow. However, under some geometric circumstances with clinical stenosis, the conversion of pressure energy to kinetic energy is correlated quite strongly to total mechanical energy loss. A relatively small amount of total energy is actually lost in the flow contraction. Most of the lost energy in flow contraction is due to viscous losses.

There may, however, be a small amount of flow separation immediately after particularly abrupt contractions. Flow separation occurs because the momentum of the flow causes it to continue to converge for a short period after the anatomic

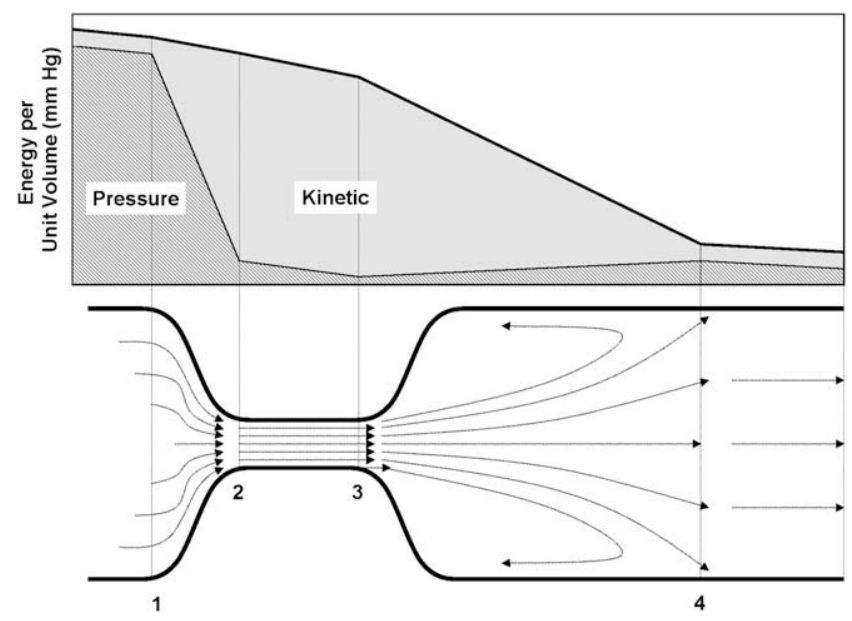

Figure 5. Energy changes in schematic arterial stenosis: Energy changes per unit volume of blood (top) and schematic arterial stenosis (bottom). contraction. Such abrupt contractions have considerably more energy loss than gradual ones.

\section{Throat section}

The second section of a stenosis is a throat of constant crosssectional area (Figure 5 -point 2 to point 3 ). The continuity equation states that the velocity at the inlet and outlet of the throat section must be the same. Therefore, there is no loss in kinetic energy; the total energy loss in this section is completely loss of static pressure. Of note, only in nonbranching vessels of constant cross-sectional area is the measured pressure gradient equivalent to total energy loss per unit volume, and usually only a small amount of total energy is lost in the throat section of a stenosis. This energy loss is viscous loss if the flow is laminar and nearly completely turbulent if the flow has enough momentum to enable its transition to turbulence.

\section{Expansion section}

A large amount of energy is lost in the expansion section of a stenosis (Figure 5-point 3 to point 4). All of this lost energy is kinetic energy, which explains why the Doppler gradient correlates so strongly with total mechanical energy loss. However, the Doppler gradient does not completely represent total energy loss because a portion of the kinetic energy is converted into pressure. This is the pressure recovery phenomenon. The expansion section illustrates why pressure gradients can be misleading in estimating the lost driving force for flow. Although the static pressure increases in the expansion section, total energy loss in this section is very large.

\section{Implications of Pathologic Energy Loss}

Energy loss is a normal feature of the circulation. The left ventricle is designed to generate the amount of energy needed to overcome these losses in the healthy circulation while moving blood at a rate necessary for normal cellular metabolism. However, pathologic conditions, such as valvular stenosis, valvular regurgitation, or aneurysm, create additional sources of energy loss. To supply blood at the necessary rate, the ventricle must perform additional work to overcome the added energy loss. To do this the ventricle must raise its systolic pressure and/or volumetric capacity. Both of these changes result in an increase in stress on the myocardium.

\section{Myocardial Response to Pressure and Volume Overload}

When the heart experiences supernormal hemodynamic pressures or volume loads, the myocardium can hypertrophy and/ or dilate. These compensatory remodeling mechanisms attempt to provide the additional work required to overcome energy losses.

Although other factors, such as, age, sex, and genetic and neurohumoral factors, can influence hypertrophy, increased mechanical stress is a sufficient stimulus to trigger 
hypertrophy. Increased load induction can affect the regulation of myocyte gene expression and lead to molecular, cellular, and interstitial changes within the myocardium. This biochemical cellular response to mechanical stimuli, known as mechanotransduction, is not well understood. ${ }^{6}$

With valvular stenosis, the additional flow impedance along the outflow tract causes ventricular pressure overload. Peak systolic pressure increases result in the myocardium experiencing higher peak systolic stresses, which act as the trigger for concentric hypertrophy, in which the ventricular wall thickens with the addition of myofibrils in parallel. The ventricle alters its shape to a more prolate spheroid, with a decrease in the short axis with respect to the long axis.

With valvular insufficiency, regurgitation imposes volume overload on the ventricle. This additional diastolic filling results in higher diastolic stresses in the myocardium, which can act as the trigger for eccentric hypertrophy, where myofibrils are added in series, and the myocytes elongate. The ventricular volume dilates and adopts a more spherical shape in the process.

Myocardial hypertrophy can occur within hours of onset of a new stimulus and can double ventricular mass within a week. In gradually worsening systolic or diastolic stresses, the hypertrophy can be insidious. The dynamic equilibrium between hypertrophy and systolic performance is a dominant theme in all stages of the clinical course of hypertrophy. In general, hypertrophy compensates for altered stresses; the additional thickness and altered geometry of concentric hypertrophy reduce peak systolic myofiber stresses, while additional capacitance and the more spherical shape of the ventricle from eccentric hypertrophy reduce diastolic fiber stresses. Hypertrophy proceeds until fiber stresses are normalized in a negative feedback system.

The feedback mechanisms for the two kinds of hypertrophy operate largely independently. With eccentric hypertrophy, a certain degree of concentric hypertrophy is also activated due to the increase in ventricular volume to mass ratio, which demands additional ventricular work. ${ }^{7}$

\section{Relation of Myocardial Changes to Energy Loss}

Increased energy loss in the circulation elicits genetic and biochemical changes in the myocardium that result in what is termed ventricular remodeling. When energy losses are initially mild, the ensuing ventricular remodeling can be considered beneficial inasmuch as it improves the performance of the myocardium. As mechanical stresses and resultant energy loss increase or as lesions persist over longer periods of time, the changes occurring with ventricular remodeling themselves can become detrimental to the heart, be they excess hypertrophy, marked dilatation, or myocardial fibrosis. The point at which remodeling transitions from beneficial to detrimental has not been well described clinically, mechanically or biochemically.
Fortunately, when mechanical stresses are relieved and energy loss returns to more normal patterns with valvular reconstruction or replacement, in many situations, such as with aortic stenosis, the detrimental ventricular remodeling can be reversed. However, in some clinical situations, such as longstanding aortic regurgitation, correction of the mechanical stresses and normalization of energy loss may not be reflected in reverse remodeling, and the detrimental changes to the myocardium can persist even after the valvular lesion is corrected.

Thus, the actual relationship of energy loss to changes imposed on the myocardium is more easily understood in the causative relationship concerning pathologic remodeling than it is in reverse remodeling when the energy loss patterns have been normalized.

\section{Current Limitations of the Use of Energy Loss}

Although pathologic energy loss is the quantity that best represents the increase in workload on the left ventricle, there are several reasons why such a loss is not routinely used in clinical evaluations. Direct calculation of energy loss is both invasive and complex. Mechanical energy losses are usually determined by measuring static pressure, velocity, and height differentials at two locations in the bloodstream. Such a calculation requires simultaneous temporal knowledge of the velocity and pressure profiles at these locations. Pressure gradient and effective orifice area, in contrast, are correlated with total energy loss and can be estimated noninvasively by Doppler echocardiography. Nonetheless, there are known parameters that can be easily and noninvasively measured and correlate better with energy loss than do pressure gradient and effective orifice area. The remainder of this article will focus on the discrepancies between the measurement of pressure gradient and effective orifice area and actual total energy loss, describe other parameters affecting total energy loss, and give some examples of how total energy loss may better be approximated in vivo for the clinical evaluation of aortic stenosis and prosthetic heart valves. The purposes of this analysis are to raise questions about current procedures, to review some newer procedural suggestions from a theoretical standpoint, and to identify gaps in current knowledge of problems in the evaluation of bulk flow hemodynamics.

\section{Energy Loss and Aortic Valve Stenosis}

\section{Evaluation of stenosis severity with pressure gradient}

The pressure gradient across a stenotic aortic valve is dependent on the flow rate across the valve. Because some patients have considerably higher or lower cardiac outputs than others, and because a patient's own cardiac output can vary markedly, the use of an isolated pressure gradient for the diagnosis of aortic stenosis severity can be misleading. There are also problems with the use of either echocardiography or catheterization to measure the pressure gradient. 
A potential problem with the use of Doppler ultrasound in the estimation of valve gradients is the phenomenon of pressure recovery. Properly placed Doppler velocity measurements will result in an overestimation of the total energy loss in aortic stenosis, and this overestimation may result in misdiagnosis. The overestimation is theoretically expected to be largest in patients with large effective orifice area/aortic cross-sectional area ratios. ${ }^{8}$ Since clinicians define increasing aortic stenosis severity by decreasing effective orifice area, ${ }^{9}$ patients most likely to suffer from misdiagnosis using Doppler gradients are those with small aortas. In these patients, the effects of pressure recovery have been shown to be pronounced. ${ }^{10}$

Another problem in the use of Doppler ultrasound for pressure gradient estimation is the placement of the transducer relative to the axis of the jet issuing from the stenosis. If the transducer is placed obliquely with respect to the jet axis, the measured velocity will underestimate the true velocity. ${ }^{11}$ Since the errors resulting from pressure recovery and oblique transducer placement can offset each other, Doppler ultrasound can at times approximate accurate pressure measurements obtained from catheterization. However, this is somewhat of a chance occurrence and should be regarded as the exception rather than the rule.

Aside from its invasive nature, the largest problem with the use of catheterization to measure pressure gradients is the placement of the catheter with respect to the valve. ${ }^{12}$ If the catheter is placed either too near to or far from the stenotic valve, it will measure only a portion of the recovered pressure and result in an overestimation of the total energy loss.

Valvular insufficiency has been observed in many patients with aortic stenosis. Such insufficiency introduces additional complications in the estimation of the degree of stenosis by standard criteria. While commonly used mean, peak, and peak-to-peak pressure gradients may correlate reasonably well with mechanical energy loss during forward flow, they have no correlation with energy loss resulting from valvular insufficiency. Since such losses can exceed those resulting from valvular stenosis, the use of forward flow pressure gradients to estimate fluid mechanical energy losses can be highly inaccurate in patients with concomitant regurgitation.

\section{Evaluation of stenosis severity with effective orifice area}

The effective orifice area is the most commonly used parameter to express the severity of aortic stenosis. Aortic stenosis can be labeled as mild, moderate, or severe ${ }^{9}$ when the effective orifice areas are more than $1.5 \mathrm{~cm}^{2}$, between 1.5 and 1.0 $\mathrm{cm}^{2}$, and less than $1.0 \mathrm{~cm}^{2}$. Effective orifice area does not vary greatly with flow rates in most patients with aortic stenosis, and, therefore, can be used as a descriptive parameter independent of cardiac output.

Many patients with an effective orifice area of $0.8 \mathrm{~cm}^{2}$ or lower, however, can be entirely asymptomatic. ${ }^{5}$ Most of the total energy lost in aortic stenosis is due to the separation involved in flow expansion. In patients with very small aortas, flow across stenotic orifices may not undergo a large expansion, thereby retaining much of its driving force for flow. Garcia and associates ${ }^{13}$ simulated aortic stenoses of the same effective orifice area but with different aortic diameters in vitro and found significant differences in energy losses among the models.

Another possible problem can arise from misinterpretation of the definition of effective orifice area. Effective orifice area does not represent the anatomic orifice area; rather it represents the cross-sectional area of the jet issuing from the valve at the point of its largest contraction, called the vena contracta. The size of the effective orifice area depends on the geometry of the contraction as well as the size of the orifice itself. For this reason, a stenotic valve with a sudden contraction may have a smaller effective orifice area than a stenotic valve with a gradual contraction, even though the two valves have identical anatomic orifice areas (Figure 6).

When catheterization is used to estimate effective orifice area (EOA), the Gorlin formula is usually applied to estimate the area of the jet at the vena contracta:

$$
E O A=\frac{Q}{44.5 \cdot C \cdot \sqrt{\Delta p}}(\text { Gorlin formula })
$$

where $Q$ is the average forward flow rate during systole, $\Delta p$ is the average mean pressure gradient across the valve during systole, and $C$ is an empirical constant, usually taken to be equal to one. ${ }^{1}$ With echocardiography, however, effective orifice area is typically estimated by applying the continuity equation to echocardiography measurements of the diameter of the ventricular outflow tract $(D)$, the temporal average of the velocity in the outflow tract during systole $\left(v_{o f t}\right)$, and the temporal average of the velocity at the vena contracta $\left(v_{v c}\right)^{14}$ :

$$
E O A=\frac{\pi D^{2} v_{o f t}}{4 v_{v c}}(\text { Continuity equation })
$$
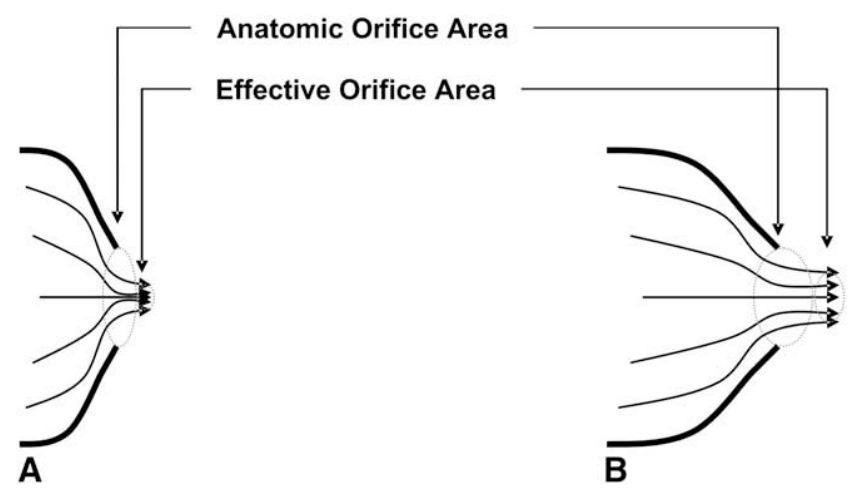

Figure 6. Differences in effective orifice area depending on abrupt contraction (A) and gradual contraction (B). 
The effective orifice area is always smaller than the anatomic orifice area. Therefore, estimations of the effective orifice area using Gorlin's formula and the continuity equation are expected to be smaller than estimations of the anatomic orifice area using length measurements made by echocardiography or magnetic resonance imaging.

In comparing the Gorlin and continuity equation representation of effective orifice area, two subtleties should be kept in mind. The first is that for an accurate estimation of the effective orifice area, the pressure gradient term in the Gorlin approach should be the Doppler gradient. As the Gorlin formula is most often used with catheter measurements, Equation 6 can be expected to overestimate effective orifice area, due to the pressure recovery phenomenon. However, this formulation, while not representing the effective orifice area, may be useful in evaluating the severity of aortic stenosis. ${ }^{10}$ The second subtlety is that all variables in Equations 6 and 7 are averages during the systolic period. Instantaneous values of pressure and flow rate cannot be used in Equation 6 unless taken at peak flow rate, because the Gorlin formula is derived using Bernoulli's equation for steady flow, which is invalid in situations where there is a large net temporal acceleration or deceleration in flow. Averages over the entire cycle may not be relevant either, especially if aortic regurgitation occurs concurrently. ${ }^{15}$ Most techniques for estimation of forward flow rate (Fick's method, thermal- and dye-dilution) are averages over the entire cardiac cycle $\left(Q_{n e t}\right)$. If no regurgitation is present, these methods can be used to estimate the average forward flow rate during systole, which is $Q$ in Equation 6. However, if aortic regurgitation is present, direct application of Gorlin's formula will result in an underestimation in effective orifice area, as:

$$
Q=\frac{Q_{n e t}}{(1-R F)}
$$

where $R F$ represents the regurgitant fraction of the aortic valve. $^{13}$

Like pressure gradient, effective orifice area is a characteristic of forward flow and cannot give information about energy losses owing to valvular insufficiency.

\section{Parameters affecting energy loss}

As described above, much more mechanical energy is lost in flow expansion than in either flow contraction or in the throat regions of arterial stenoses. The localization of energy loss to the flow expansion region is more pronounced in aortic valve stenosis than in arterial stenosis owing to the gradual nature of the flow contraction, the short length of the throat, and the abruptness of flow expansion in aortic valve stenosis. Total energy loss in aortic valve stenosis occurs almost completely in the expansion section. ${ }^{16}$ This simplifies the problem considerably by allowing energy losses occurring in aortic valve stenosis to be modeled by an abrupt expansion.
The total energy loss per unit volume across an abrupt expansion (see Appendix) can be rewritten as the following for the situation of aortic valve stenosis:

$$
\Phi_{a s}=\frac{1}{2} \rho v_{v c}\left(1-\left(\frac{E O A}{A_{\text {aorta }}}\right)\right)^{2}
$$

where $\Phi_{a s}$ is energy loss, $v_{v c}$ is the temporal average of the velocity at the vena contracta and $\rho$ is the density of blood. Energy loss over an aortic valve stenosis is a strong function of the effective orifice area $(E O A)$ of the valve and the crosssectional area of the aorta $\left(A_{\text {aorta }}\right)$. As this area ratio approaches zero, the energy loss per unit volume in the expansion approaches the Doppler gradient. Thus, in cases of severe aortic valve stenosis, the Doppler gradient is equivalent to mechanical energy loss per unit volume. The Doppler gradient is worst in estimating the recovered pressure gradients in patients with small aortas because the area ratio is further from zero in these patients than in others. The cross-sectional area of the expansion should be taken as the cross-sectional area of the aorta at the sinotubular junction, as the separated flow disappears after the flow has traversed the sinuses of Valsalva. ${ }^{17}$

Geometric changes in the aortic inflow tract resulting from left ventricular hypertrophy can lead to further energy losses. In severe hypertrophy, blood may approach the valve from up to $45^{\circ}$ as it is turned around the protruding wall of the ventricular septum. ${ }^{18}$ Subaortic obstruction has been observed to constrict the outflow tract diameter to a length as small as $6 \mathrm{~mm} .{ }^{19}$ Subaortic obstruction, in particular, has been identified with substantial pressure gradients, and concomitant surgical removal of such obstruction has been recommended when replacing the aortic valve. ${ }^{20}$

In addition to energy loss from forward flow across the aortic valve, there is energy loss from leakage when the valve is insufficient. The regurgitant flow rate $\left(Q_{\text {reg }}\right)$ is a function of the regurgitant orifice area $(R O A)$ and the pressure difference $(\Delta p)$ across the closed valve. These three quantities are related in a way similar to that of how forward flow rate, pressure difference across the open valve, and the effective orifice area are related by Gorlin's formula. ${ }^{21}$

$$
R O A=\frac{Q_{\text {reg }}}{44.5 \cdot C \cdot \sqrt{\Delta p}}
$$

\section{Examples of more effective energy loss evaluation}

Accurate clinical determination of total energy loss in all clinical settings has not been elucidated to date. However, several groups have used various estimates of energy loss to try to better define the severity of aortic stenosis and its natural history. 
In 2003, Bermejo and associates ${ }^{22}$ used several methods, including peak jet velocity, mean pressure gradient, left ventricular stroke work loss, aortic valve area, and aortic valve resistance, to assess aortic stenosis clinically and to evaluate them as predictors of outcome, such as symptoms, risk of a late event, and all cause mortality. Of the indices tested, the best predictor of all outcomes investigated was left ventricular stroke work loss. That concept [defined as: $100 \cdot \Delta \mathrm{P} /(\Delta \mathrm{P}+\mathrm{SPB})$, where $\Delta \mathrm{P}$ is mean transvalvular systolic pressure gradient and SPB is systolic blood pressure], and which was expressed as a percentage, was defined decades ago by Tobin and colleagues. ${ }^{23}$

Heinrich and coworkers ${ }^{5}$ showed both analytically and through in vitro experiments that the ratio of effective orifice area to aortic cross-sectional area was correlated with energy loss. As this ratio decreased below a value of 0.2 , total energy losses increase in an exponential manner. The clinical validity of this approach had been made years earlier by $\mathrm{Oh}$ and coworkers ${ }^{24}$ who suggested that the left ventricular outflow tract velocity/stenotic orifice velocity ratio be used as a measure of the severity of aortic valve stenosis. The area and velocity ratios can be shown by continuity to be equivalent if the cross-sectional area of the left ventricular outflow tract is equivalent to the cross-sectional area of the aorta. Oh and coworkers found that a velocity ratio lower than 0.25 had a high correlation with symptoms of severe aortic stenosis in patients.

The commonly used indexed effective orifice area (effective orifice area indexed to body surface area) suggested by Dumesnil and Yoganathan ${ }^{25}$ may evaluate total energy loss as well as the ratio of the effective orifice area to aortic cross-sectional area. This is likely due to the close correlation between the aortic cross-sectional area and the patient's body surface area. However, if this correlation is invalid for a particular patient, the ability of this index to estimate stenosis severity will be compromised. Tongue and associates ${ }^{26}$ have showed a strong correlation between the body indexed effective orifice area and symptoms of aortic stenosis severity in patients.

In 2003, Garcia and coworkers ${ }^{10}$ described what they termed the energy loss coefficient to evaluate the severity of aortic stenosis. This coefficient is defined by Gorlin's formula, either using the recovered pressure gradient instead of the Doppler gradient or by using the effective orifice area and echocardiographic measurements of aortic diameter. The energy loss coefficient does not represent an actual area but instead describes the relationship between flow rate and total energy loss across the aortic valve. Garcia's group demonstrated a strong correlation between the energy loss coefficient obtained from catheter measurements and from Doppler measurements in vitro, in animals, and in patients. Tongue and colleagues ${ }^{26}$ showed a strong correlation between the energy loss coefficient and symptoms of aortic stenosis severity in patients.
Recently, Garcia, Pibarot, and Durand ${ }^{16}$ presented a means of calculating instantaneous energy loss per unit volume across the aortic valve in an explicit fashion, based only on effective orifice area, aortic cross-sectional area, flow rate, and the change in flow rate with respect to time. This method was accurate in controlled in vitro tests and may hold some promise in future clinical use.

Energy loss owing to regurgitant flow must be considered in patients with concomitant valvular insufficiency. To our knowledge, no clinical studies of energy loss resulting from aortic valve insufficiency have been made, but some studies have assessed mitral regurgitation. MacIsaac and associates ${ }^{27}$ studied energy transfer from the left ventricle to the left atrium in patients with severe mitral regurgitation. They divided this transfer into two forms: kinetic energy and potential energy (static pressure) transfer. The kinetic energy transfer, sometimes referred to as "left atrial kinetic energy," can be estimated by echocardiography, whereas potential energy transfer could only be estimated indirectly. Theoretically, all kinetic energy transferred across the incompetent valve should be lost, whereas some potential energy transferred may be used to drive forward flow again. Teien and colleagues ${ }^{28}$ found a strong correlation between the left atrial kinetic energy and increases in ventricular work in porcine animal models. They stated that the left atrial kinetic energy is at least as important a determinant of ventricular work as regurgitant volume.

An estimation of such energy loss per unit volume net forward flow owing to regurgitant flow can be made by assuming that regurgitant flow rate is constant over the diastolic period and changes in gravitational and kinetic energy are negligible with respect to changes in pressure energy. Under these conditions,

$$
\Phi_{\text {reg }}=\Delta p \cdot R F
$$

where $\Phi_{\text {reg }}$ is energy loss, $\Delta p$ is the average pressure gradient across the closed valve during diastole, and $R F$ is the regurgitant fraction. The total energy loss caused by a stenotic, insufficient valve is equivalent to the sum of forward and regurgitant flow energy loss, which allows a potential method of evaluation of the impact of mixed valve disease on ventricular function.

\section{Prosthetic Heart Valve Performance Evaluation of prosthetic valve performance with pressure gradient}

Like pressure gradients across native aortic valve stenoses, pressure gradients across prosthetic valves depend on the flow rate across the valve. However, unlike in the clinical evaluation of native aortic stenosis, evaluation of prosthetic valve performance is usually performed with a standard for comparison between pressure drop and flow rate. Pressure gradient is therefore a useful tool in the evaluation of 
prosthetic heart valve performance in forward flow. For clinical measurement of pressure gradient in patients with Doppler echocardiography, however, the problems of pressure recovery and measurement at an oblique angle are problematic. Pressure recovery can be expected to cause problems in the estimation of pressure gradient in patients with prosthetic valves because these valves usually present with only a small degree of stenosis.

Additional complications arise in using pressure gradient to evaluate the total energy loss across mechanical prostheses. The first problem is that considerable difference between Doppler and catheter gradients may exist, which does not result from pressure recovery, because mechanical prostheses have more than one orifice and each orifice may offer a different resistance to flow. Velocity measurements made in the one orifice are not necessarily representative of the gradient in either pressure or total energy as flow traverses the valve. This is a particular problem with bileaflet prostheses ${ }^{29}$ because the central orifice of most current designs of these prostheses creates considerably more resistance to flow than the two lateral orifices. ${ }^{30}$ The difference between Doppler and catheter gradients has been so notable for mechanical prostheses that empirically derived correction factors, dependent on specific valve design, have been suggested to the Food and Drug Administration for the correlations between the measured Doppler gradient and the desired pressure gradient. ${ }^{31}$ Some studies claim a negligible difference between pressure gradients measured with catheter and Doppler, particularly with regard to mean gradients. ${ }^{32}$ However, because of its potential problems, the use of Doppler to evaluate the performance of mechanical prostheses should be approached with care, and Doppler measurements from the same orifice are desirable if they are to be compared.

Another problem with using pressure gradients to evaluate total energy loss across mechanical prostheses is that nearly all mechanical prostheses used today have some leakage incorporated into their design. Pressure gradient information is useful to evaluate the forward flow energy loss of prosthetic valves after they are implanted. However, when choosing a valve based on its ability to assist in the movement of blood through the circulation, energy loss from leakage as well as from forward flow should be considered. Energy loss from leakage flow is not very large compared with forward flow energy loss in small aortic valves, but it can be greater than energy loss from forward flow in larger prostheses, particularly those in the mitral position. ${ }^{33}$

\section{Evaluation of prosthetic valve performance with effective orifice area}

Like pressure gradients, prosthetic valve effective orifice areas can only be correlated with forward flow energy loss. Since much of the energy loss in mitral prostheses can come from leakage, the effective orifice area may not be the best criterion to judge the suitability of large mechanical implants for the mitral position. Estimations of the effective orifice area from the continuity equation (Equation 4) have the same multiple orifice problem as Doppler gradient measurements, as Equation 4 is used with echocardiography.

\section{Parameters affecting energy loss}

During forward flow, total energy loss across prosthetic heart valves is a function of valve type, size, orientation, and position. Of currently implanted valves, stentless bioprostheses offer the least resistance to flow, followed by bileaflet mechanical designs, tilting disc designs, and stented bioprostheses, as tested in vitro. ${ }^{34}$ Larger valves cause less resistance to forward flow than smaller valves. To limit energy loss, one should avoid tilting the housing of the valve relative to the annulus to maximize the effective orifice area, ${ }^{35}$ and orient the occluder of the valve parallel to the incoming forward flow when the occluder is in the fully open position to minimize drag. ${ }^{36}$ Forward flow energy losses are particularly problematic in aortic valve replacement in small patients. ${ }^{37}$

Energy loss over closed prosthetic valves during leakage flow is governed by valve type, size, and position. Bioprostheses do not leak if functioning properly. Bileaflet mechanical designs tend to have more energy loss owing to leakage than tilting disc designs. ${ }^{33}$ Larger valves leak more than smaller ones. Regurgitant energy loss is most problematic in positions where the pressure gradient during closure and leakage is high and where the duration of leakage is long. For example, these losses can exceed forward flow energy losses in large mitral prostheses. ${ }^{33}$

The presence of paravalvular leak, calcification or tearing of bioprosthesis tissue, structural dysfunction of mechanical components, or formation of pannus or thrombus at or near the orifice may increase energy loss after valve prosthesis implantation. The effects of these events on energy loss can be very high if either the opening or the closing of the valve is impeded.

\section{Examples of more effective energy loss evaluation}

Total energy losses over the cardiac cycle can be accurately estimated in vitro, inasmuch as flow, pressure, and velocity can be measured simultaneously at several locations. Although measured pressure gradients and estimated effective orifice areas can have strong correlations with forward flow energy loss, they give no information during the leakage phase, when the valve is closed. Leakage flow represents blood that must be pumped again through the valve, an additional source of energy loss. When choosing a valvular prosthesis for implantation, it would be preferable to use the sum of forward flow and leakage energy losses, rather than isolated pressure gradient or effective orifice area information, as a standard in the comparison of hemodynamics. This is most important in the implantation of larger prostheses or mitral prostheses. Energy losses between different prostheses can only be compared when evaluated under 
similar and physiologically relevant flow and pressure conditions.

For the clinical evaluation of prosthetic valve performance in a particular patient, methods for the estimation of energy loss more effective than the use of pressure gradient and echocardiography to investigate possible problems in opening and leakage have yet to be thoroughly investigated. However, accurate methods for evaluation of energy loss in native aortic valve stenosis are likely applicable to the clinical evaluation of the energy loss caused by bioprostheses placed in the aortic and pulmonary positions of a patient. This is due to the similarity in geometry between aortic stenosis and these prosthetic designs. Each of these cases involves a single orifice opening abruptly into a tubular expansion.

\section{Summary}

Methods describing the severity of native heart valve lesions and functional performance of prosthetic heart valves have not kept pace with available technologies, clinical methods of evaluation, or derived indices of valve performance that represent their impact on ventricular function. Although the concept of energy loss has been understood for many years, its use was hampered by difficulty in obtaining the clinical performance measurements needed to calculate energy loss. Noninvasive clinical techniques to measure the quantities needed to estimate energy loss have become universally available and widely used but have not been associated with an advance in the derived measurement of energy loss, the most important estimate of the impact aspect of native and prosthetic valve function on left ventricular function. We believe the time has come for clinicians and researchers to move past rudimentary measurements of valve systolic function and focus on the effect of total valve performance on heart function, namely energy loss. It is not the valve; it is the ventricle that ought to be the focus of evaluation.

\section{References}

1. Gorlin R, Gorlin SG. Hydraulic formula for calculation of the stenotic mitral valve, other cardiac valves, and central circulatory shunts. Am Heart J. 1951;41:1-29.

2. Starr A, Edwards ML. Mitral replacement: clinical experience with a ball-valve prosthesis. Ann Surg. 1961;154:726-40.

3. Harken DE, Soroff HS, Taylor WJ, Lefemine AA, Gupta SK, Lunzer S. Partial and complete prostheses in aortic insufficiency. J Thorac Cardiovasc Surg. 1960;40:744-62.

4. Leefe SE, Gentle CR. Theoretical evaluation of energy loss methods in the analysis of prosthetic heart valves. J Biomed Eng. 1987;9:121-7.

5. Heinrich RS, Marcus RH, Ensley AE, Gibson DE, Yoganathan AP. Valve orifice area alone is an insufficient index of aortic stenosis severity: effects of the proximal and distal geometry on transaortic energy loss. J Heart Valve Dis. 1999;8:509-15.

6. Colan SD. Ventricular function in volume overload lesions. In: Fogel MA, ed. Ventricular function and blood flow in congenital heart disease. Philadelphia: Blackwell; 2005:205-22.

7. Colan SD. Ventricular function in pressure overload lesions. In: Fogel MA, ed. Ventricular function and blood flow in congenital heart disease. Philadelphia: Blackwell; 2005:187-204.

8. Baumgartner H, Stefenelli T, Niederberger J, Schima H, Maurer G. "Overestimation" of catheter gradients by Doppler ultrasound in pa- tients with aortic stenosis: a predictable manifestation of pressure recovery. J Am Coll Cardiol. 1999;33:1655-61.

9. Bonow RO, Carabello B, Chatterjee K, de Leon AC Jr, Faxon DP, Freed MD, et al. . ACC/AHA 2006 Guidelines for the management of patients with valvular heart disease. A report of the American College of Cardiology/American Heart Association Task Force on Practice Guidelines. Circulation. 2006;114:e84-231.

10. Garcia D, Dumesnil JG, Durand L, Kadem L, Pibarot P. Discrepancies between catheter and Doppler estimates of valve effective orifice area can be predicted from the pressure recovery phenomenon. $J$ Am Coll Cardiol. 2003;41:435-42.

11. Yoganathan AP, Recusani F, Valdez-Cruz L, Sung HW, Sahn DJ. Oblique flow vectors from dispersing jets produce the velocity overestimation on angle corrected continuous wave Doppler studies: in vitro laser Doppler investigations. Circulation. 1987;76(Suppl IV):355.

12. Voelker W, Reul H, Stelzer T, Schmidt A, Karsch K. Pressure recovery in aortic stenosis: an in vitro study in a pulsatile flow model. J Am Coll Cardiol. 1992;20:1585-93.

13. Garcia D, Pibarot P, Dumesnil JG, Sakr F, Durand LG. Assessment of aortic valve stenosis severity: a new index based on the energy loss concept. Circulation. 2000;101:765-71.

14. Zoghbi WA, Farmer KL, Soto JG, Nelson JG, Quinones MA. Accurate non-invasive quantification of stenotic aortic valve area by Doppler echocardiography. Circulation. 1986;73:452-9.

15. Scotten L, Walker D, Dutton J. Modified Gorlin equation for the diagnosis of mixed aortic valve pathology. J Heart Valve Dis. 2002;11:360-8.

16. Garcia D, Pibarot P, Durand L. Analytical modelling of the instantaneous pressure gradient across the aortic valve. J Biomech. 2005;38: 1303-11.

17. Gjertsson P, Caidahl K, Svensson G, Wallentin I, Bech-Hanssen O. Important pressure recovery in patients with aortic stenosis and high Doppler gradients. Am J Cardiol. 2001;88:139-44.

18. Gross-Sawicka E, Pringle T, Lipworth B. Aortoseptal angulation and left ventricular hypertrophy pattern: an echocardiographic study in patients with aortic valve stenosis. J Am Soc Echocardiogr. 1991;4:583-8.

19. White R, Obuchowski N, Gunawardena S, Lipchik E, Lever H, Van Dyke C, et al. Aortic inflow tract obstruction in hypertrophic cardiomyopathy: presurgical and postsurgical evaluation by computed tomography magnetic resonance imaging. Am J Card Imaging. 1996;10:1-13.

20. Come P, Pringle T, Lipworth B. Doppler evidence that true left ventricular to aortic pressure gradients exist in hypertrophic cardiomyopathy. Am Heart J. 1988;5:1253-61.

21. Askenazi J, Carlson CJ, Alpert JS, Dexter L. Mitral valve area in combined mitral stenosis and regurgitation. Circulation. 1976;54:480-3.

22. Bermejo J, Edreman R, Feijoo J, Moreno MM, Gomez-Moreno P, Garcia-Fernendez MA. Clinical efficacy of Doppler-echocardiographic indices of aortic valve stenosis: a comparative test-based analysis of outcome. J Am Coll Cardiol. 2003;41:142-51.

23. Tobin JR, Rahimtoola SH, Blundell PE, Swan HJ. Percentage of left ventricular stroke work loss. A simple hemodynamic concept for estimation of severity in valvular aortic stenosis. Circulation. 1967;35:868-79.

24. Oh JK, Taliercio CP, Holmes DR, Reeder GS, Bailey KR, Steward JB, et al. Prediction of the severity of aortic stenosis by Doppler aortic valve area determination: prospective Doppler catheterization correlation in 100 patients. J Am Coll Cardiol. 1988;11:1227-34.

25. Dumesnil JG, Yoganathan AP. Valve prosthesis hemodynamics and the problem of high transprosthetic pressure gradients. Eur J Cardiothorac Surg. 1992;6(Suppl 1):S34-8.

26. Tongue AG, Dumesnil JG, Laforest I, Theriault C, Durand LG, Pibarot P. Left ventricular longitudinal shortening in patients with aortic stenosis: relationship with symptomatic status. J Heart Valve Dis. 2003; $12: 142-9$

27. MacIsaac AI, McDonald IG, Kirsner RL, Graham SA, Tanzar D. Left ventricular energy in mitral regurgitation: a preliminary report. Aust NZ J Med. 1992;22(Suppl 5):532-40.

28. Teien DE, Jones M, Shiota T, Yamada I, Frithiof D, Sahn DJ. Left ventricular stroke work in mitral regurgitation: an animal experimental study. J Heart Valve Dis. 1997;6:613-20.

29. Mascherbauer J, Schima H, Maurer G, Baumgartner H. Doppler assessment of mechanical aortic valve prostheses: effect of valve design and size of the aorta. J Heart Valve Dis. 2004;13:823-30. 
30. Travis BR, Leo HL, Shah PA, Frakes DH, Yoganathan AP. An analysis of turbulent shear stresses in leakage flow through a bileaflet mechanical prostheses. J Biomech Eng. 2002;124:155-65.

31. Stewart SF, Herman BA, Nell DM, Retta SM. Effects of valve characteristics on the accuracy of the Bernoulli equation: a survey of data submitted to the U.S. FDA. J Heart Valve Dis. 2004;13:461-6.

32. Knebel F, Gliech V, Walde T, Eddicks S, Konertz W, Baumann G, et al. High concordance of invasive and echocardiographic mean pressure gradients in patients with a mechanical aortic valve prosthesis. $J$ Heart Valve Dis. 2005;14:332-7.

33. Strüber M, Campbell A, Richard G, Laas J. Hydrodynamic function of tilting disc prostheses and bileaflet valves in double valve replacement. Eur J Cardiothorac Surg. 1996;10:422-7.
34. Yoganathan AP, Travis BR. Fluid dynamics of prosthetic valves. In: Otto C, ed. The practice of clinical echocardiography. 2nd ed. Philadelphia: WB Saunders; 2002:50-524.

35. Hartrumph M, Albes JM, Krempl T, Rudolph V, Wahlers T. The hemodynamic performance of a standard bileaflet valve is impaired by a tilted implantation position. Eur J Cardiothorac Surg. 2003;23:283-91.

36. Travis BR, Heinrich RS, Ensley AE, Gibson DE, Hashim S, Yoganathan AP. The hemodynamic effects of mechanical prosthetic valve type and orientation on fluid mechanical energy loss and pressure drop in in vitro models of ventricular hypertrophy. J Heart Valve Dis. 1998;7:345-54.

37. Fisher J. Comparative study of the hydrodynamic function of six size 19 mm bileaflet heart valves. Eur J Cardiothorac Surg. 1995;9:692-6.

\section{Appendix: Mathematical Explanations}

\section{Bernoulli's Equation Corrected for Energy Loss}

Bernoulli's equation for steady flow can be corrected by adding a term $(\Phi)$ to account for energy loss per unit volume occurring between points 1 and 2 .

$$
\frac{1}{2} \rho v_{1}^{2}+\rho g h_{1}+p_{1}=\frac{1}{2} \rho v_{2}^{2}+\rho g h_{2}+p_{2}+\Phi
$$

Thus the energy loss per unit volume can be expressed as:

$$
\Phi=\left(p_{1}-p_{2}\right)+\frac{1}{2} \rho\left(v_{1}^{2}-v_{2}^{2}\right)+\rho g\left(h_{1}-h_{2}\right)
$$

\section{Physical Explanations of Energy Loss}

Reynolds number. The Reynolds number can be described as an approximation to the ratio of inertial to frictional forces in a flow. The inertial force of a moving flow is the force required to bring the flow to rest, whereas the frictional force of such a flow is created by viscous shear stress acting on solid surfaces. In vessel flow, the Reynolds number is often expressed as:

$$
N_{\mathrm{Re}}=\frac{D \bar{v} \rho}{\mu}
$$

where $D$ is the inner diameter of the vessel, $v$ is the average velocity of the flow, $\rho$ is the fluid density, and $\mu$ is the fluid viscosity.

Viscous losses. An example of viscous losses is represented by the pressure loss across a horizontal, straight vessel under steady, laminar flow. Under these conditions, gravitational and kinetic energy in the flow do not change over the length of the vessel, and Equation A2 becomes:

$$
\Phi=p_{1}-p_{2}
$$

Under steady flow, there is always a balance in the vessel between pressure forces acting on the cross-sectional area of the inlet and outlet of the vessel and the viscous shear stress on the vessel walls. Pressure forces result from pressure acting on the cross-sectional area of the inlet and outlet of the vessel, and shear forces result from shear stress acting on the area of the inner vessel wall. The force balance can therefore be represented as:

$$
\frac{1}{4} \pi D^{2} p_{1}-\frac{1}{4} \pi D^{2} p_{2}=\pi D L \tau_{w}
$$

where $D$ is the inner diameter of the vessel, $L$ is the vessel length, $p_{1}$ is the pressure at the entrance to the vessel, $p_{2}$ is the pressure at the exit of the vessel, and $\tau_{w}$ is the shear stress on the vessel walls. Combining Equations A2 and A3, energy loss can be defined as:

$$
\Phi=\frac{4 L \tau_{w}}{D}
$$

Under laminar flow conditions, where only viscous energy loss is present, the ratio of kinetic energy per unit volume in the flow to shear stress on the vessel walls is analogous to the ratio of inertial to frictional forces in the flow, the quantity which is approximated by the Reynolds number. In laminar flow through a circular vessel, ratio of kinetic energy per unit volume in the flow to shear stress on the vessel walls differs from the Reynolds number by a numerical factor of 16 :

$$
\frac{\frac{1}{2} \rho \bar{v}^{2}}{\tau_{w}}=\frac{N_{\mathrm{Re}}}{16}=\frac{1}{16} \frac{\rho \bar{v} D}{\mu}
$$

where $\rho$ is the fluid density, $v$ the average velocity, and $\mu$ the fluid viscosity. Combining Equations A6 and A7:

$$
\Phi=\frac{32 L \mu \bar{v}}{D^{2}}
$$

If the flow rate:

$$
Q=\frac{1}{4} \pi D^{2} \bar{v}
$$

is substituted for average velocity, Equation A8 becomes:

$$
\Phi=\frac{128}{\pi} \frac{Q \mu L}{D^{4}}
$$


Equation A10 shows that viscous energy losses in this geometry are inversely proportional to the fourth power of the vessel diameter, which is why such losses decrease very quickly with small increases in vessel radius. This equation also shows that viscous energy losses are directly proportional to the flow rate, vessel length, and fluid viscosity.

Turbulent losses. Equations A4 to A6 are also valid for the energy loss across a horizontal, straight vessel under fully turbulent flow. The ratio of kinetic energy per unit volume in the flow to shear stress on the vessel walls, however, is more of a function of the roughness of the vessel wall than the Reynolds number. This is characterized by an empirically determined factor $f$, which is a function primarily of surface roughness and has a small dependence on Reynolds number:

$$
\frac{\frac{1}{2} \rho \bar{v}^{2}}{\tau_{w}}=\frac{1}{f}
$$

Combining Equations A11 and A6,

$$
\Phi=\frac{2 L \rho \bar{v}^{2} f}{D}
$$

Combining Equations A12 and A9 to express Equation A12 in terms of flow rate:

$$
\Phi=\frac{32 f}{\pi^{2}} \frac{Q^{2} \rho L}{D^{5}}
$$

Typical values of $f$ for turbulent flow in vessels do not vary considerably with Reynolds number. Taking $f$ to be approximately constant for a given vessel, Equation A13 shows that turbulent losses are dependent on fluid density and have only small viscosity dependence. This can be expected, inasmuch as turbulence is initiated by changes in fluid momentum rather than viscous shear. Turbulent losses are in general proportional to the square of the flow rate, which is also demonstrated by Equation A13.

Flow separation losses. An example of flow separation losses is represented by a sudden expansion of flow from a horizontal vessel of cross-sectional area $A_{1}$ to vessel of cross-sectional area $A_{2}$. Under these conditions, gravitational energy in the flow does not change over the length of the vessel, and Equation A2 becomes:

$$
\Phi=\left(p_{1}-p_{2}\right)+\frac{1}{2} \rho\left(v_{1}^{2}-v_{2}^{2}\right)
$$

Newton's second law states that any change in momentum of the flow must be accompanied by a force. In this case, the change in momentum of the flow as it travels from point 1 to point 2 must be caused by a difference in the pressure acting on the cross-sectional area of the inlet and outlet of the vessel. This is the source of the pressure recovery phenomenon:

$$
p_{1} A_{2}-p_{2} A_{2}=m\left(v_{2}-v_{1}\right)
$$

where $m$ is the mass flow rate and $A_{1}$ and $A_{2}$ are the cross-sectional areas at points 1 and 2, respectively. It is worth noting that in an abrupt flow expansion such as that shown in Figure A2, the pressure at point 1 acts on the downstream area $A_{2}$. Combining Equations A14 and A15,

$$
\Phi=m \frac{v_{2}-v_{1}}{A_{2}}+\frac{1}{2} \rho\left(v_{1}^{2}-v_{2}^{2}\right)
$$

The mass flow rate can be rewritten in terms of the fluid density and the velocity and cross-sectional area at the vessel inlet:

$$
m=\rho v_{1} A_{1}
$$

Combining Equations A16 and A17,

$$
\Phi=\rho v_{1} A_{1} \frac{v_{2}-v_{1}}{A_{2}}+\frac{1}{2} \rho\left(v_{1}^{2}-v_{2}^{2}\right)
$$

The downstream velocity can then be expressed in terms of the upstream velocity by the continuity equation:

$$
v_{2}=v_{1} \frac{A_{1}}{A_{2}}
$$

Combining Equations A18 and A19:

$$
\Phi=\frac{1}{2} \rho v_{1}^{2}\left(1-\frac{A_{1}}{A_{2}}\right)^{2}
$$

Combining Equations A9 and A20:

$$
\Phi=\frac{8}{\pi^{2}} \frac{Q^{2} \rho}{D_{1}^{4}}\left(1-\left(\frac{D_{1}}{D_{2}}\right)^{2}\right)^{2}
$$

Equation A21 shows that flow separation losses, like turbulent losses, are initiated by changes in fluid momentum rather than viscous shear. Such losses have no viscosity dependence and are proportional to the fluid density and the square of the flow rate. Equation A20 also shows that flow separation losses are heavily dependant on the ratio of cross-sectional areas between the smaller and larger vessels. 